\title{
Jet shape resummation using soft-collinear effective theory
}

\author{
Yang-Ting Chien and Ivan Vitev \\ Theoretical Division, T-2, Los Alamos National Laboratory, \\ Los Alamos, NM 87545, U.S.A. \\ E-mail: ytchien@lanl.gov, ivitev@lanl.gov
}

ABSTRACT: The jet shape is a classic jet substructure observable that probes the average transverse energy profile inside a reconstructed jet. The studies of jet shapes in protonproton collisions have served as precision tests of perturbative Quantum Chromodynamics (QCD). They have also recently become the baseline for studying the in-medium modification of parton showers in ultra-relativistic nucleus-nucleus collisions. The jet shape is a function of two angular parameters $R$ and $r$, which can be at hierarchical scales. Its calculation suffers from large logarithms of the ratio between the two scales, and these phase space logarithms can be conveniently resummed in the framework of soft-collinear effective theory (SCET). We find that, up to power corrections, the integral jet shape can be expressed in a factorized form which involves only the ratio between two jet energy functions. Resummation is performed at next-to-leading logarithmic order using renormalization-group evolution techniques. Comparisons to jet shape measurements at the Large Hadron Collider (LHC) are presented to verify the dominant role of the collinear parton shower and to identify the kinematic region in which power-suppressed soft modes and non-perturbative effects may play a role.

KeYwords: QCD Phenomenology, Jets

ARXIV EPRINT: 1405.4293 


\section{Contents}

1 Introduction $\quad 1$

2 The observable $\quad 4$

3 Factorized expression for the jet shape $\quad 5$

3.1 One-loop jet energy functions 9

$\begin{array}{lll}3.2 & \text { Renormalization-group equations and resummation } & 12\end{array}$

4 Results 14

$\begin{array}{llr}5 & \text { Summary and discussion } & 18\end{array}$

\section{Introduction}

Studies of jet substructure provide precision tests of perturbative QCD in high energy processes. They originate from the studies of event shapes in $e^{+} e^{-}$collisions, which helped test and confirm the gauge theory structure of QCD [1-9]. Recently, accurate event shape calculations also allowed one of the most precise extraction of the strong coupling constant [10-13]. However, at hadron colliders, due to the presence of beam remnants, underlying event and pileup, the studies of event-wide inclusive observables become much more complicated. Instead, investigation of exclusive jet substructure observables attracts more attention, and considerable progress has been made in this direction [14]. One of the goal of such studies is to help distinguish possible signals of new physics beyond the Standard Model from large QCD backgrounds. An important problem, for example, is to develop improved methods to distinguish quark-initiated from gluon-initiated jets $[15,16]$. Advances in this area will have numerous applications in new physics searches.

Among the observables instrumental in quark-gluon discrimination, a classic jet substructure observable called the jet shape [17] has been studied for more than two decades. The integral jet shape is the average fraction of the transverse energy of the jet measured within a subcone of size $r$, smaller than the size $R$ of the jet, around the jet axis which is conventionally chosen to be along the 3 -momentum of the jet. The differential jet shape is then the derivative with respect to $r$. The jet shape probes the transverse energy distribution inside a jet, which is very different for quark-initiated and gluon-initiated jets. Typically, quark jets are more localized whereas gluon jets are more spread out. This can be seen from the locations of the peaks of the differential jet shape distributions in figure 1 , shown here as an illustration. Historically, the jet shape was introduced and calculated in QCD at leading-order in [17]. The observable was later resummed using the modified leading logarithmic approximation [18]. The contributions from initial state radiation and 
non-perturbative effects were also examined. A phenomenological parameter $R_{\text {sep }}[17,18]$ can be used to fit the data with the leading-order results. For more precise comparison with the experimental data a next-to-leading order calculation is required, but the result is not available at this time. Refs. $[19,20]$ give another resummation framework using perturbative QCD and a comparison with the Tevatron and the LHC data.

On the other hand, the studies of jet shapes in heavy ion collisions have drawn considerable attention in the high-energy nuclear physics community. One of the top priorities of the heavy ion program at the LHC is to determine the properties of the hot, dense medium which is produced in ultra-relativistic nuclear collisions and referred to as the quark-gluon plasma (QGP). In such highly energetic collisions of ions, jets are produced and subsequently quenched as they propagate through the medium [21, 22]. The jet quenching phenomena give strong evidence for the creation of the QGP [23-25] and build upon the well-established leading particle suppression pattern. The modification of jet shapes provides unique information about the structure of the in-medium parton showers. The first measurement of the modification of jet shapes in lead-lead $(\mathrm{Pb}+\mathrm{Pb})$ collisions with small experimental uncertainties was performed by the CMS collaboration $[26,27]$. On the theory side, ref. [21] builds upon the jet shape calculations in proton-proton collisions [18] and studies the medium modification of jet shapes using the Gyulassy-Levai-Vitev formalism $[28,29]$ in the soft gluon limit. Monte Carlo transport simulations of jet shapes in $\mathrm{Pb}+\mathrm{Pb}$ collisions at the $\mathrm{LHC}$ have also been recently performed [30, 31]. However, a study going beyond these approximations and addressing the precision of the jet shape calculations in a systematically improvable way is needed in both proton-proton and heavy ion collisions.

In this paper, we focus on the jet shape calculations in proton-proton collisions using soft-collinear effective theory (SCET) [32-36]. The calculations in heavy ion collisions using SCET with Glauber gluon interactions in the medium [37, 38], and the full mediuminduced splitting functions [39, 40] and applications [41] will be discussed in a forthcoming paper [42]. SCET is an effective field theory of QCD for processes with energetic lightlike and soft degrees of freedom with a systematic power counting. In events with highly collimated jets, the power counting parameter $\lambda \sim m_{J} / Q$, which is the ratio between the jet mass and the jet energy, is small and the leading power contribution calculated in SCET is a very good approximation of the full QCD result. SCET separates physics at different energy scales, and the factorization of the hard, collinear and soft sectors is more transparent. The hard, jet and soft functions involved in the factorization theorem of a physical cross section, as well as their anomalous dimensions, can be calculated order by order at each characteristic scale. Large logarithms of the ratio between hierarchical energy scales are resummed through the renormalization-group evolution of these functions.

In the context of jet shape calculations, as we will see below, for small radii $R$ the observable depends mostly on the jet energy and the partonic origin of the jet. It is not sensitive to the details of the underlying hard scattering processes as well as the soft radiation in the whole event. The contribution from the soft radiation to the jet shape is power suppressed. Therefore, the collinear sector is dominant in such calculations and the factorized expression for the jet shape involves only the ratio between two jet energy 

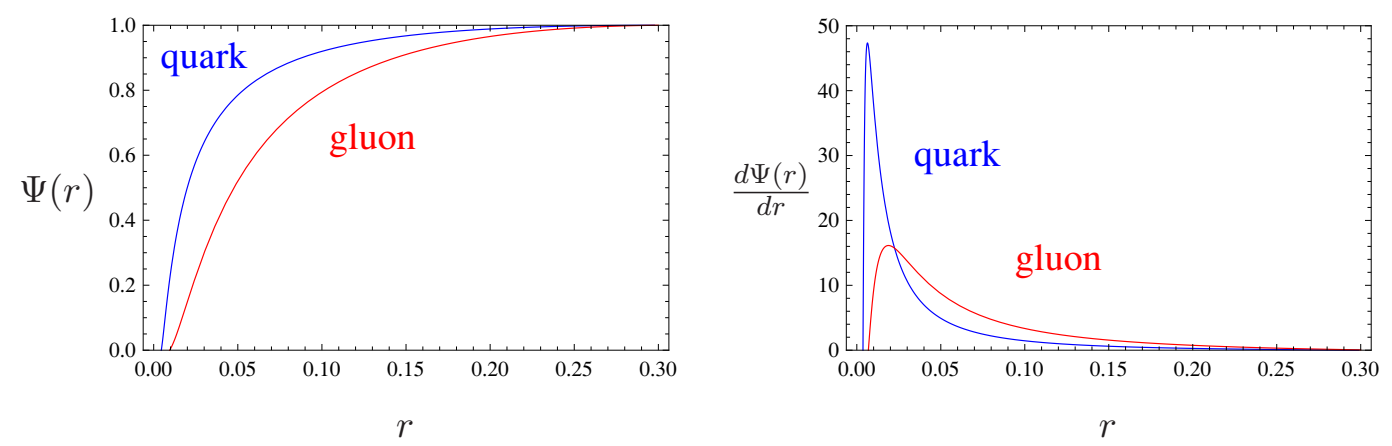

Figure 1. The integral (left) and differential (right) jet shapes of quark and gluon jets of size $R=0.3$ in proton-proton collisions, plotted as an illustration of their differences. Jet shape contains the information about the transverse energy distribution inside a jet. On average, quark jets are more localized whereas gluon jets are more spread out.

functions, which we define and calculate at leading order (LO). We then identify two characteristic jet scales, each proportional to the angular scale $(r$ or $R)$ within which we measure the transverse energy. The logarithms of the ratio between the two jet scales are exactly the logarithms of the form $\ln r / R$, which we can resum through the renormalizationgroup evolution of the jet energy functions between the two jet scales. With the two-loop cusp anomalous dimension, the two-loop running of the strong coupling constant and the one-loop anomalous dimension of the jet energy function, the jet shape is resummed to next-to-leading logarithmic (NLL) accuracy. ${ }^{1}$ However, in this work we do not include the contributions from initial state radiation and non-perturbative effects. We ignore power suppressed terms of $\mathcal{O}(R)$ and focus on the resummation of large logarithms. Note that terms of $\mathcal{O}(r / R)$ can still be large at $r \approx R$ and they are properly captured by the SCET formalism. To systematically extend the precision to next-to-leading order (NLO) and next-to-next-to leading logarithmic (NNLL) accuracy, we will need the two-loop jet energy function and its two-loop anomalous dimension. At this order the issue about non-global logarithms [44] and the way to resum them will also arise. We will leave these interesting topics for future work.

The rest of the paper is organized as follows. In section 2 we give the definition of the jet shape and discuss the choice of the jet axis, which is related to the form of the factorized expression. In section 3 we discuss the power counting and the calculation of the jet shape in SCET. We show that the contribution from soft particles to the jet shape is power suppressed if we choose a soft-recoil free axis $[45,46]$ in the jet shape definition. The factorized expression of the jet shape has a simple product form without recoil-momentum convolution. This leads to the cancellation of the hard and soft functions in the factorized

\footnotetext{
${ }^{1} \mathrm{By} \mathrm{N} \mathrm{N}^{\mathrm{k}} \mathrm{LL}$ we mean the resummed series includes terms of the form $\alpha_{s}^{n} \ln ^{m} r / R$ with $2 n \geq m \geq 2 n-2 k+1$. We use this convention in the region of validity of $r$ where $\alpha_{s} \ln ^{2} r / R \lesssim 1$, which is the case in comparing with both the CMS and ATLAS measurements, as we will see. However, in the region where $\alpha_{s} \ln r / R \sim$ 1 , more terms should be resummed using the convention which is commonly referred to as counting in the exponent. [43] gives a useful discussion about the counting of precisions in both perturbative QCD and SCET calculations. Note that non-global logarithms affect the $\alpha_{s}^{2} \ln ^{2} r / R$ terms at NNLL in the convention above.
} 
expression for the jet shape, which involves only the ratio of the jet energy functions. We also give the operator definition of the jet energy function and calculate it at one-loop for both quark and gluon jets reconstructed using the cone or the anti- $\mathrm{k}_{\mathrm{T}}$ algorithm [47]. The resummation of jet shapes is performed to NLL accuracy using the renormalizationgroup techniques, and we estimate the uncertainties of our calculations by varying the characteristic jet scales. In section 4 we compare our resummed jet shape results with the PYTHIA 8 simulations and the CMS measurements. We present our conclusion and give an outlook in section 5 .

\section{The observable}

In this section we will give the jet shape definition and discuss some of the related subtleties. Before we can study any property of a jet, we need to precisely define what a jet is. This is conventionally done using a jet algorithm with a parameter $R$, which characterizes the size of the jet. Different jet algorithms will give jets with different substructures. For a jet reconstructed using a jet algorithm, we first define a jet axis $\hat{n}$. A natural choice of $\hat{n}$ is the direction of the 3 -momentum of the jet. However, such axis is not necessarily along the direction of the dominant energy flow within the jet. The factorized expression for an observable referencing this axis will involve an intricate convolution over the recoil momentum between the collinear and the soft sectors. On the other hand, there are choices of $\hat{n}$, e.g. the broadening axis or the winner-take-all axis $[45,46]$, which are soft-recoil free. These axes absorb the recoil sensitivity and point along the collinear momentum which gives a simpler factorized form without recoil-momentum convolution. We will come back to this point in more details in section 3 .

Given a jet with an axis $\hat{n}$, its integral jet shape $\Psi_{J}(r)$ is defined as follows,

$$
\Psi_{J}(r)=\frac{\sum_{i, d_{i \hat{n}}<r} E_{T}^{i}}{\sum_{i, d_{i \hat{n}}<R} E_{T}^{i}},
$$

which is the fraction of the transverse energy $E_{T}$ of the jet within an angular scale $r$ from the jet axis. The transverse energy is measured with respect to the beam direction. By definition $\Psi_{J}(R)=1$. Here, $d_{i \hat{n}}$ is the distance metric between the $i$-th particle in the jet and the jet axis $\hat{n}$. It can be the Euclidean distance between the two directions along the particle and the jet axis on the rapidity-azimuthal angle $(y, \phi)$ plane,

$$
d_{i \hat{n}}=\sqrt{\left(y_{i}-y_{\text {jet }}\right)^{2}+\left(\phi_{i}-\phi_{\text {jet }}\right)^{2}} .
$$

Note that, with this definition the jet shape is boost invariant along the beam direction. So we can calculate the jet shape in the frame where the jet is central $\left(y_{\text {jet }}=0\right)$. In that frame, up to power corrections, the metric is equivalent to

$$
d_{i \hat{n}}=\cos ^{-1}\left(\hat{n}_{i} \cdot \hat{n}\right)
$$

which is the angle between the momentum of the $i$-th particle and the jet axis in 3 -space. Also, up to power corrections we can consider the energies instead of the transverse energies 
of particles in the central jet. So, in this paper,

$$
\Psi_{J}(r)=\frac{\sum_{i, \theta_{i \hat{n}}<r} E^{i}}{\sum_{i, \theta_{i \hat{n}}<R} E^{i}} \equiv \frac{E_{r}}{E_{R}},
$$

setting $y_{\text {jet }}=0$. In experiment, we measure the averaged integral jet shape $\Psi(r)$ (we will drop the word averaged and refer to $\Psi(r)$ as the integral jet shape from now on),

$$
\Psi(r)=\frac{1}{N_{J}} \sum_{J=1}^{N_{J}} \Psi_{J}(r) .
$$

The differential jet shape $\psi(r)$ is defined to be its derivative,

$$
\psi(r)=\frac{d \Psi(r)}{d r},
$$

which tells us how the energy inside the jet is distributed in $r$. Recall that the jet shape has dependence on the jet algorithm and the parameter $R$ used in the jet definition, which we suppress here for notational simplicity. At the Tevatron mostly the iterative cone algorithm was used, whereas at the LHC the anti- $\mathrm{k}_{\mathrm{T}}$ algorithm is used almost exclusively.

The jet shape is a function of $r$ and $R$, which are parameters or angular coordinates. In the jet shape calculations terms of the form $\alpha_{s}^{n} \ln ^{m} r / R$ appear, which can become large if $r \ll R$. In this regime the fixed order expansion breaks down and the large logarithms need to be resummed. The resummation was performed some time ago using the modified leading logarithmic approximation (MLLA) [18], including the contributions from initial state radiation and non-perturbative effects. A phenomenological parameter $R_{\text {sep }}$, which can be thought of as the effective separation between the particles at leading order, can be used to fit with the experimental data. In this paper we will not follow this phenomenological approach.

In the next section we will calculate the jet shape using SCET. Large logarithms come from the presence of multiple, hierarchical energy scales in the problem. This is the situation in which effective field theory techniques are useful because the corrections to the leading power contribution are suppressed by a small power counting parameter. The key ingredient in this approach is the factorization, which separates physics into multiple, single-scaled sectors. Large logarithms are then resummed by the renormalization-group evolution of different pieces of physics between their natural scales.

\section{Factorized expression for the jet shape}

For concreteness, let us consider the shapes of jets from an $N$-jet configuration in $e^{+} e^{-}$ collisions without loss of generality, as we will see. ${ }^{2}$ Jets are reconstructed using either the cone or the anti- $\mathrm{k}_{\mathrm{T}}$ algorithm with a parameter $R$ that is parametrically small. At the

\footnotetext{
${ }^{2}$ The $N$ jets are assumed to be energetic by passing a hard $p_{T}$ cut so that the power counting and the factorized expression we will write down are valid. At hadron colliders, there are power-suppressed contributions of $\mathcal{O}(R)$ from initial state radiation which we will neglect.
} 


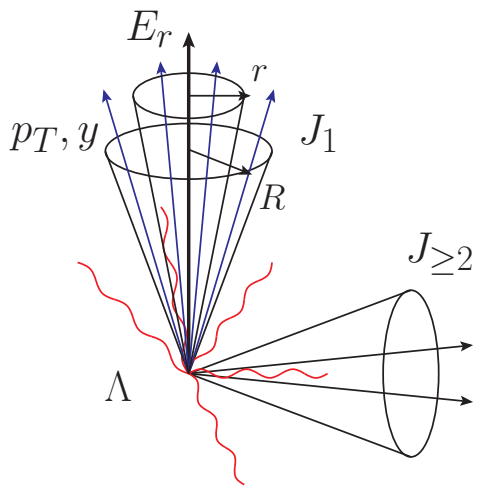

Figure 2. Schematic event topology of $N$-jet production with collinear and soft radiation. Jets are reconstructed using a jet algorithm with a parameter $R$. The energy $E_{r}$ inside a cone of size $r$ in $J_{1}$ is measured, as well as its transverse momentum $p_{T}$ and rapidity $y$. An energy cutoff $\Lambda$ outside the jets is imposed to ensure the $N$-jet configuration.

LHC a typical $R=0.5$ to 0.7 is chosen in the physics analyses of proton-proton collisions. For heavy ion collisions, because of the underlying event contaminations a even smaller $R=0.3$ is chosen at CMS. In these cases jets are highly collimated and the process can be accurately described by the soft-collinear effective theory (SCET). An energy cutoff $\Lambda$ outside the jets is imposed to ensure an $N$-jet configuration. We also measure the energy $E_{r}$ inside a cone of size $r$ in one jet (labeled by 1 ), as well as the transverse momenta $p_{T i}$ and pseudo-rapidity $y_{i}$ of all the jets.

Before we write down the factorized expression for the jet shape in SCET, let us study the power counting of the observable. In light-cone coordinates with $p=\left(\bar{n} \cdot p, n \cdot p, \vec{p}_{\perp}\right)$ where $n=(1, \hat{n})$ and $\bar{n}=(1,-\hat{n})$, the momentum scalings of the collinear and the soft particles are

$$
p_{c}=Q\left(1, \lambda^{2}, \lambda\right), p_{s}=Q(\lambda, \lambda, \lambda)
$$

where $Q$ is the center of mass energy of the $e^{+} e^{-}$collisions and $\lambda$ is the power counting parameter which describes how wide a jet is spread out. There is one collinear sector for for each jet with the collinear direction $n$. The power counting parameter satisfies $R \gtrsim \lambda$ so that most of the jet energy is included in the jet reconstruction. The jet energy $E$ has contributions $E^{c}$ and $E^{s}$ from both the collinear and the soft sectors,

$$
\Psi_{J}(r)=\frac{E_{r}}{E_{R}}=\frac{E_{r}^{c}+E_{r}^{s}}{E_{R}^{c}+E_{R}^{s}}=\frac{E_{r}^{c}}{E_{R}^{c}}+\mathcal{O}(\lambda)
$$

Up to power corrections, the integral jet shape can be calculated using only the collinear momenta, and the soft contributions can be neglected. This approximation works best when $R$ is not large. On the other hand, as was briefly discussed in section 2 , soft radiation can potentially alter the collinear momentum direction by an $\mathcal{O}(\lambda)$ amount. For $r$ of $\mathcal{O}(\lambda)$ or smaller, soft recoil can actually change $E_{r}$ by an $\mathcal{O}(1)$ amount which will spoil the simple factorized expression. This can be remedied by choosing the recoil-free jet axes for the 
jet shape measurements. ${ }^{3}$ Such jet axes will always point along the collinear momentum directions $[45,46]$. To avoid the issue of recoil from the soft particles outside the jets, we impose a constraint on the energy cutoff $\Lambda$ by demanding $\Lambda / Q \ll R$. At hadron colliders, because of dynamical threshold enhancement the partonic phase space where the jets have small jet masses dominates in the cross section calculations [48-51]. The cross section of jet configurations with large jet masses is suppressed because parton distribution functions die off quickly when the momentum fraction of the parton is close to one. This will reduce the recoil sensitivity of the jet axis and therefore it is less of an issue.

Detailed derivations and discussions about the factorized expression similar to the one we will write down here can be found in $[46,52,53]$. The differential cross section for $N$-jet production with jets $p_{T_{i}}$ and $y_{i}$, an energy $E_{r}$ inside the cone of size $r$ in one jet (labeled by 1 ), and an energy cutoff $\Lambda$ outside all the jets is the following, ${ }^{4}$

$$
\begin{aligned}
\frac{1}{\sigma_{0}} \frac{d \sigma}{d E_{r} d p_{T_{i}} d y_{i}}= & H\left(p_{T_{i}}, y_{i}, \mu\right) J_{\omega_{1}}\left(E_{r}, \mu\right) J_{\omega_{2}}(\mu) \ldots J_{\omega_{N}}(\mu) S_{n_{1} n_{2} \ldots n_{N}}(\Lambda, \mu) \\
& +\mathcal{O}\left(\frac{\Lambda}{Q}\right)+\mathcal{O}(R) .
\end{aligned}
$$

Here, $H\left(p_{T_{i}}, y_{i}, \mu\right)$ is the hard function which contains the information about the $N$-jet productions at high scale $Q$ and is independent of the jet shape measurements. It is the square of the Wilson coefficient when we match QCD and SCET at the hard scale. $J_{\omega}\left(E_{r}, \mu\right)$ is a newly defined jet function, which is the probability of measuring an energy $E_{r}$ inside a $r$ cone for a jet of size $R$ with $\omega=2 E_{J}=2 p_{T} \cosh y$,

$$
J_{\omega}\left(E_{r}, \mu\right)=\sum_{X_{c}}\left\langle 0\left|\bar{\chi}_{\omega}(0)\right| X_{c}\right\rangle\left\langle X_{c}\left|\chi_{\omega}(0)\right| 0\right\rangle \delta\left(E_{r}-\hat{E}^{<r}\left(X_{c}\right)\right) .
$$

Here, $\chi_{\omega}$ is the collinear jet field in SCET, and the operator $\hat{E}^{<r}$ returns the energy of the collinear particles $X_{c}$ inside the $r$ cone. By imposing an energy cutoff $\Lambda$ outside the jets, we are essentially restricting the collinear radiation to be all inside the jets up to corrections of $\mathcal{O}(\Lambda / Q)$. All the other jet functions without the jet energy measurements are the "unmeasured" jet functions [53]. Once we integrate out the collinear modes, we are left with a soft sector which is described by soft Wilson lines along the jet directions. The soft function is defined as follows,

$$
S_{n_{1} n_{2} \ldots n_{N}}(\Lambda, \mu)=\sum_{X_{s}}\left\langle 0\left|\mathcal{O}_{s}^{\dagger}(0)\right| X_{s}\right\rangle\left\langle X_{s}\left|\mathcal{O}_{s}(0)\right| 0\right\rangle \Theta\left(\Lambda-\hat{E}^{>R}\left(X_{s}\right)\right)
$$

where $\mathcal{O}_{s}(0)$ consists of $N$ soft Wilson lines along the $n_{1,2, \ldots, N}$ directions intersecting at the origin 0 . The operator $\hat{E}^{>R}$ returns the energy of the soft particles $X_{s}$ outside all $N$ jets. Note that the factorized form is a simple product of the hard, jet and soft functions without any convolution. This is because the soft particles don't contribute to the jet energy at

\footnotetext{
${ }^{3}$ This has not been implemented in experimental measurements to-date, and it introduces another power correction when comparing our calculations with experiments.

${ }^{4}$ The functional dependence on $r$ and $R$ are suppressed for notational simplicity.
} 
leading power and we choose a recoil-free jet axis in the jet shape definition. All the jet and soft functions have $R$ dependence which we suppress for brevity.

Similarly, for the differential jet rate of $N$-jet production,

$$
\frac{1}{\sigma_{0}} \frac{d \sigma}{d p_{T_{i}} d y_{i}}=H\left(p_{T_{i}}, y_{i}, \mu\right) J_{\omega_{1}}(\mu) J_{\omega_{2}}(\mu) \ldots J_{\omega_{N}}(\mu) S_{n_{1} n_{2} \ldots n_{N}}(\Lambda, \mu)+\mathcal{O}\left(\frac{\Lambda}{Q}\right)+\mathcal{O}(R)
$$

with the same hard, unmeasured jet (from 2 to $N$ ) and soft functions. The only difference is that we don't measure the energy of jet 1 , so $J_{\omega_{1}}\left(E_{r}, \mu\right)$ is replaced by the unmeasured jet function $J_{\omega_{1}}(\mu)$. Now, the averaged energy inside the cone of size $r$ in jet 1 with $\omega=\omega_{1}$ is

$$
\begin{aligned}
\left\langle E_{r}\right\rangle_{\omega_{1}} & =\frac{\int d E_{r} E_{r} \frac{1}{\sigma_{0}} \frac{d \sigma}{d E_{r} d p_{T_{i}} d y_{i}}}{\frac{1}{\sigma_{0}} \frac{d \sigma}{d p_{T_{i}} d y_{i}}}=\frac{H\left(p_{T_{i}}, y_{i}, \mu\right) J_{\omega_{1}}^{E_{r}}(\mu) J_{\omega_{2}}(\mu) \ldots J_{\omega_{N}}(\mu) S_{n_{1} n_{2} \ldots n_{N}}(\Lambda, \mu)}{H\left(p_{T_{i}}, y_{i}, \mu\right) J_{\omega_{1}}(\mu) J_{\omega_{2}}(\mu) \ldots J_{\omega_{N}}(\mu) S_{n_{1} n_{2} \ldots n_{N}}(\Lambda, \mu)} \\
& =\frac{J_{\omega_{1}}^{E_{r}}(\mu)}{J_{\omega_{1}}(\mu)} .
\end{aligned}
$$

Here,

$$
J_{\omega}^{E_{r}}(\mu)=\int d E_{r} E_{r} J_{\omega}\left(E_{r}, \mu\right)
$$

is referred to as the jet energy function. Note that all the hard, unmeasured jet, and soft functions cancel in the calculations because of the product form of the factorized expression. The integral jet shape needs another average over the jet production cross sections, with proper phase space $(P S)$ cuts on $p_{T}$ and $y$, and is therefore

$$
\Psi(r)=\frac{1}{\sigma_{\text {total }}} \sum_{i=q, g} \int_{P S} d p_{T} d y \frac{d \sigma^{i}}{d p_{T} d y} \Psi_{\omega}^{i}(r),
$$

where

$$
\Psi_{\omega}(r)=\frac{\left\langle E_{r}\right\rangle_{\omega}}{\left\langle E_{R}\right\rangle_{\omega}}=\frac{J_{\omega}^{E_{r}}(\mu) / J_{\omega}(\mu)}{J_{\omega}^{E_{R}}(\mu) / J_{\omega}(\mu)}=\frac{J_{\omega}^{E_{r}}(\mu)}{J_{\omega}^{E_{R}}(\mu)},
$$

which is the ratio between two jet energy functions, and $\omega=2 p_{T}$ in the frame where the jet is central $(y=0)$. As we can see, the jet shape does not depend on the hard and soft functions nor on the information about the other jets. In other words, it is insensitive to the hard process and the soft radiation and depends only on the partonic origin and the energy of the jet.

From the factorized form we can already infer some non-trivial properties of the jet and soft functions. By the renormalization-group invariance of the physical cross sections, the anomalous dimension of the soft function should be independent of the energy cutoff $\Lambda$ to all orders in perturbation theory. Therefore the soft anomalous dimension can only be a function of $R$ and is independent of the renormalization scale $\mu$. Also, the anomalous dimension of the jet energy function $J_{\omega}^{E_{r}}(\mu)$ is the same as the anomalous dimension of the unmeasured jet function $J_{\omega}(\mu)$ which does not depend on $r$. This makes $\Psi_{\omega}(r)$ renormalization-group invariant. Furthermore, the $R$ dependence in the anomalous dimensions of the jet and soft functions has to cancel because the hard function does not depend on $R$. 


\subsection{One-loop jet energy functions}

Having set up the factorization framework, now we proceed to calculate the jet energy function $J_{\omega}^{E_{r}}(\mu)$ at $\mathcal{O}\left(\alpha_{s}\right)$ for both quark jets and gluon jets reconstructed using the cone or anti- $\mathrm{k}_{\mathrm{T}}$ algorithm. At this order, the initial collinear particle with momentum $l$ splits into two collinear particles with momenta $q$ and $l-q$. We use lightcone coordinates throughout the calculations where $k^{+} \equiv n \cdot k$ and $k^{-} \equiv \bar{n} \cdot k$. Also, we use dimensional regularization to regulate the divergences with the spacetime dimension $d=4-2 \epsilon$, and the $\overline{\mathrm{MS}}$ renormalization scheme. For quark jets,

$$
\begin{aligned}
J_{\omega, \text { alg }}^{q E_{r}}(\mu)= & 4 \pi \alpha_{s}\left(\frac{\mu^{2} e^{\gamma_{E}}}{4 \pi}\right)^{\epsilon} C_{F} \int \frac{d l^{+}}{2 \pi} \frac{1}{\left(l^{+}\right)^{2}} \int \frac{d^{d} q}{(2 \pi)^{d}}\left[4 \frac{l^{+}}{q^{-}}+(d-2) \frac{l^{+}-q^{+}}{\omega-q^{-}}\right] \\
& \times 2 \pi \delta\left(q^{-} q^{+}-q_{\perp}^{2}\right) \Theta\left(q^{-}\right) \Theta\left(q^{+}\right) 2 \pi \delta\left(l^{+}-q^{+}-\frac{q_{\perp}^{2}}{\omega-q^{-}}\right) \Theta\left(\omega-q^{-}\right) \Theta\left(l^{+}-q^{+}\right) \\
& \times\left(\mathcal{M}_{1}+\mathcal{M}_{2}+\mathcal{M}_{3}+\mathcal{M}_{4}\right)
\end{aligned}
$$

and for gluon jets,

$$
\begin{aligned}
J_{\omega, \text { alg }}^{g E_{r}}(\mu)= & 8 \pi \alpha_{s}\left(\frac{\mu^{2} e^{\gamma_{E}}}{4 \pi}\right)^{\epsilon} \int \frac{d l^{+}}{2 \pi} \frac{1}{l^{+}} \int \frac{d^{d} q}{(2 \pi)^{d}}\left[T_{F} n_{f}\left(1-\frac{2}{1-\epsilon} \frac{q^{+} q^{-}}{\omega l^{+}}\right)\right. \\
& \left.-C_{A}\left(2-\frac{\omega}{q^{-}}-\frac{\omega}{\omega-q^{-}}-\frac{q^{+} q^{-}}{\omega l^{+}}\right)\right] 2 \pi \delta\left(q^{2}\right) \Theta\left(q^{-}\right) \Theta\left(q^{+}\right) \\
& \times 2 \pi \delta\left((l-q)^{2}\right) \Theta\left(\omega-q^{-}\right) \Theta\left(l^{+}-q^{+}\right) \times\left(\mathcal{M}_{1}+\mathcal{M}_{2}+\mathcal{M}_{3}+\mathcal{M}_{4}\right) .
\end{aligned}
$$

Here

$$
\begin{aligned}
& \mathcal{M}_{1}=\Theta\left(\tan ^{2} \frac{r}{2}-\frac{q^{+}}{q^{-}}\right) \Theta\left(\tan ^{2} \frac{r}{2}-\frac{l^{+}-q^{+}}{\omega-q^{-}}\right) \Theta_{\mathrm{alg}} \times l^{0} \\
& \mathcal{M}_{2}=\Theta\left(\tan ^{2} \frac{r}{2}-\frac{q^{+}}{q^{-}}\right) \Theta\left(\frac{l^{+}-q^{+}}{\omega-q^{-}}-\tan ^{2} \frac{r}{2}\right) \Theta_{\mathrm{alg}} \times q^{0} \\
& \mathcal{M}_{3}=\Theta\left(\frac{q^{+}}{q^{-}}-\tan ^{2} \frac{r}{2}\right) \Theta\left(\tan ^{2} \frac{r}{2}-\frac{l^{+}-q^{+}}{\omega-q^{-}}\right) \Theta_{\mathrm{alg}} \times\left(l^{0}-q^{0}\right) \\
& \mathcal{M}_{4}=\Theta\left(\frac{q^{+}}{q^{-}}-\tan ^{2} \frac{r}{2}\right) \Theta\left(\frac{l^{+}-q^{+}}{\omega-q^{-}}-\tan ^{2} \frac{r}{2}\right) \Theta_{\mathrm{alg}} \times 0,
\end{aligned}
$$

which are the cases where each of the two particles are either inside the cone of size $r$ or not. The algorithm dependence enters in the calculations through $\Theta_{\text {alg }}$ in the following way,

$$
\begin{aligned}
\Theta_{\text {cone }} & =\Theta\left(\tan ^{2} \frac{R}{2}-\frac{q^{+}}{q^{-}}\right) \Theta\left(\tan ^{2} \frac{R}{2}-\frac{l^{+}-q^{+}}{\omega-q^{-}}\right) \\
\Theta_{\mathrm{k}_{\mathrm{T}}} & =\Theta\left(\tan ^{2} \frac{R}{2}-\frac{q^{+} \omega^{2}}{q^{-}\left(\omega-q^{-}\right)^{2}}\right) .
\end{aligned}
$$

Note that at this order the phase space constraint is the same for anti- $\mathrm{k}_{\mathrm{T}}$ jets and $\mathrm{k}_{\mathrm{T}}$ jets, so we label the anti- $\mathrm{k}_{\mathrm{T}}$ jet energy functions by $\mathrm{k}_{\mathrm{T}}$ for simplicity. For cone jets the two collinear particles are constrained to be inside a cone of size $R$, whereas for anti- $\mathrm{k}_{\mathrm{T}}$ jets the 
angle between the two collinear particles has to be smaller than $R$. Therefore one expect that cone jets are more spread out than anti- $\mathrm{k}_{\mathrm{T}}$ jets.

We expand the dimensionally regularized one-loop jet energy functions in series of $\epsilon$ 's. The anomalous dimension can be extracted from the coefficient of the $1 / \epsilon$ pole in the series, and the $\mathcal{O}\left(\epsilon^{0}\right)$ piece will be the renormalized jet energy function in the $\overline{\mathrm{MS}}$ scheme. The jet energy functions for cone jets at $\mathcal{O}\left(\alpha_{s}\right)$ are

$$
\begin{aligned}
\frac{2}{\omega} J_{\omega, \text { cone }}^{q E_{r}}(\mu)=\frac{\alpha_{s} C_{F}}{2 \pi} & {\left[\frac{1}{2} \ln ^{2} \frac{\omega^{2} \tan ^{2} \frac{r}{2}}{\mu^{2}}-\frac{3}{2} \ln \frac{\omega^{2} \tan ^{2} \frac{r}{2}}{\mu^{2}}-2 \ln X \ln \frac{\omega^{2} \tan ^{2} \frac{r}{2}}{\mu^{2}}\right.} \\
+ & 2-\frac{3 \pi^{2}}{4}+4 \operatorname{Li}_{2}\left(\frac{X}{1+X}\right)+3 \ln (1+X)+\frac{3 X}{1+X}+2 \ln ^{2}(1+X) \\
+ & \left.\left(-5 \ln (1+X)+\frac{5 X+2 X^{2}}{1+X}-2 X^{2} \ln \frac{X}{1+X}\right) \tan ^{2} \frac{R}{2}\right],
\end{aligned}
$$

and

$$
\begin{aligned}
\frac{2}{\omega} J_{\omega, \text { cone }}^{g E_{r}}(\mu)= & \frac{\alpha_{s}}{2 \pi}\left[\frac{C_{A}}{2} \ln ^{2} \frac{\omega^{2} \tan ^{2} \frac{r}{2}}{\mu^{2}}-\left(\frac{11}{6} C_{A}-\frac{2}{3} T_{F} n_{f}\right) \ln \frac{\omega^{2} \tan ^{2} \frac{r}{2}}{\mu^{2}}\right. \\
& -2 C_{A} \ln X \ln \frac{\omega^{2} \tan ^{2} \frac{r}{2}}{\mu^{2}}-\left(\frac{5 \pi^{2}}{12}-2 \operatorname{Li}_{2}\left(\frac{X}{1+X}\right)+2 \operatorname{Li}_{2}\left(\frac{1}{1+X}\right)\right) C_{A} \\
& +\left(\frac{11}{3} C_{A}-\frac{4}{3} T_{F} n_{f}+2 C_{A} \ln X\right) \log (1+X) \\
& +\frac{-2\left(5+63 X+81 X^{2}+35 X^{3}\right) T_{F} n_{f}+\left(65+351 X+477 X^{2}+203 X^{3}\right) C_{A}}{36(1+X)^{3}} \\
& -\left(\frac{2 X\left(6+13 X+9 X^{2}\right) T_{F} n_{f}-X\left(36+85 X+63 X^{2}+12 X^{3}\right) C_{A}}{6(1+X)^{3}}\right. \\
& \left.\left.+2 C_{A} X^{2} \ln X-2\left(T_{F} n_{f}-C_{A}\left(3-X^{2}\right)\right) \ln (1+X)\right) \tan ^{2} \frac{R}{2}\right]
\end{aligned}
$$

where

$$
X=\frac{\tan \frac{r}{2}}{\tan \frac{R}{2}} \approx \frac{r}{R} \quad \text { for } r, R \ll 1,
$$

is boost-invariant along the jet direction. In fact, under a Lorentz boost with rapidity $\beta$ along the jet direction, $\omega$ and $r$ transform in the following way,

$$
\omega \rightarrow e^{\beta} \omega, \tan \frac{r}{2} \rightarrow e^{-\beta} \tan \frac{r}{2} .
$$

Therefore the combination, $\omega \tan \frac{r}{2}$, is also boost-invariant. For anti- $\mathrm{k}_{\mathrm{T}}$ jets, we have

$$
\begin{aligned}
\frac{2}{\omega} J_{\omega, \mathrm{k}_{\mathrm{T}}}^{q E_{r}}(\mu)=\frac{\alpha_{s} C_{F}}{2 \pi} & {\left[\frac{1}{2} \ln ^{2} \frac{\omega^{2} \tan ^{2} \frac{r}{2}}{\mu^{2}}-\frac{3}{2} \ln \frac{\omega^{2} \tan ^{2} \frac{r}{2}}{\mu^{2}}-2 \ln X \ln \frac{\omega^{2} \tan ^{2} \frac{r}{2}}{\mu^{2}}\right.} \\
+ & \left.-\frac{3 \pi^{2}}{4}+6 X-\frac{3}{2} X^{2}-\left(\frac{1}{2} X^{2}-2 X^{3}+\frac{3}{4} X^{4}+2 X^{2} \ln X\right) \tan ^{2} \frac{R}{2}\right],
\end{aligned}
$$


and

$$
\begin{aligned}
\frac{2}{\omega} J_{\omega, \mathrm{k}_{\mathrm{T}}}^{g E_{r}}(\mu)=\frac{\alpha_{s}}{2 \pi}[ & \frac{C_{A}}{2} \ln ^{2} \frac{\omega^{2} \tan ^{2} \frac{r}{2}}{\mu^{2}}-\left(\frac{11}{6} C_{A}-\frac{2}{3} T_{F} n_{f}\right) \ln \frac{\omega^{2} \tan ^{2} \frac{r}{2}}{\mu^{2}} \\
& -2 C_{A} \ln X \ln \frac{\omega^{2} \tan ^{2} \frac{r}{2}}{\mu^{2}}+\left(\frac{65}{36}-\frac{3 \pi^{2}}{4}+8 X-3 X^{2}+\frac{8 X^{3}}{9}-\frac{X^{4}}{4}\right) C_{A} \\
& +\left(-\frac{5}{18}-4 X+3 X^{2}-\frac{16 X^{3}}{9}+\frac{X^{4}}{2}\right) T_{F} n_{f} \\
& -\frac{X^{2}}{30}\left(\left(25-80 X+45 X^{2}-16 X^{3}+5 X^{4}+60 \ln X\right) C_{A}\right. \\
& \left.\left.+\left(-20+40 X-45 X^{2}+32 X^{3}-10 X^{4}\right) T_{F} n_{f}\right) \tan ^{2} \frac{R}{2}\right] .
\end{aligned}
$$

An important observation is that the choice of the renormalization scale $\mu=\omega \tan \frac{r}{2}$ eliminates all the large logarithms at $\mathcal{O}\left(\alpha_{s}\right)$. Also, the terms from the second line down in each expression of the jet energy function are non-singular when $X \rightarrow 0$. The jet energy functions for jets reconstructed using different algorithms differ by these non-singular terms at this order. For $r=R$, we have

$$
\begin{aligned}
& \frac{2}{\omega} J_{\omega, \text { cone }}^{q E_{R}}(\mu)=J_{\omega, \text { cone }}^{q}(\mu)+\frac{\alpha_{s}}{2 \pi} C_{F}\left(\frac{7}{2}-3 \ln 2\right) \tan ^{2} \frac{R}{2} \\
& \frac{2}{\omega} J_{\omega, \text { cone }}^{g E_{R}}(\mu)=J_{\omega, \text { cone }}^{g}(\mu)+\frac{\alpha_{s}}{2 \pi}\left[C_{A}\left(\frac{49}{12}-4 \ln 2\right)-T_{F} n_{f}\left(\frac{7}{6}-2 \ln 2\right)\right] \tan ^{2} \frac{R}{2},
\end{aligned}
$$

and

$$
\begin{aligned}
& \frac{2}{\omega} J_{\omega, \mathrm{k}_{\mathrm{T}}}^{q E_{R}}(\mu)=J_{\omega, \mathrm{k}_{\mathrm{T}}}^{q}(\mu)+\frac{\alpha_{s}}{2 \pi} \frac{3}{4} C_{F} \tan ^{2} \frac{R}{2} \\
& \frac{2}{\omega} J_{\omega, \mathrm{k}_{\mathrm{T}}}^{g E_{R}}(\mu)=J_{\omega, \mathrm{k}_{\mathrm{T}}}^{g}(\mu)+\frac{\alpha_{s}}{2 \pi}\left[\frac{7}{10} C_{A}+\frac{1}{10} T_{F} n_{f}\right] \tan ^{2} \frac{R}{2} .
\end{aligned}
$$

Here

$$
\begin{aligned}
& J_{\omega, \text { alg }}^{q}(\mu)=\frac{\alpha_{s}}{2 \pi}\left(-\frac{3}{2} C_{F} \ln \frac{\omega^{2} \tan ^{2} \frac{R}{2}}{\mu^{2}}+\frac{1}{2} C_{F} \ln ^{2} \frac{\omega^{2} \tan ^{2} \frac{R}{2}}{\mu^{2}}+d_{\mathrm{alg}}^{q}\right) \\
& J_{\omega, \text { alg }}^{g}(\mu)=\frac{\alpha_{s}}{2 \pi}\left(-\frac{1}{2} \beta_{0} \ln \frac{\omega^{2} \tan ^{2} \frac{R}{2}}{\mu^{2}}+\frac{1}{2} C_{A} \ln ^{2} \frac{\omega^{2} \tan ^{2} \frac{R}{2}}{\mu^{2}}+d_{\mathrm{alg}}^{g}\right),
\end{aligned}
$$

are the unmeasured jet functions [53], with $\beta_{0}=\frac{11}{3} C_{A}-\frac{4}{3} T_{F} n_{f}$ and

$$
\begin{aligned}
d_{\mathrm{cone}}^{q} & =C_{F}\left(\frac{7}{2}+3 \ln 2-\frac{5 \pi^{2}}{12}\right) \\
d_{\mathrm{k}_{\mathrm{T}}}^{q} & =C_{F}\left(\frac{13}{2}-\frac{3 \pi^{2}}{4}\right) \\
d_{\mathrm{cone}}^{g} & =C_{A}\left(\frac{137}{36}+\frac{11}{3} \ln 2-\frac{5 \pi^{2}}{12}\right)-T_{F} n_{f}\left(\frac{23}{18}+\frac{4}{3} \ln 2\right) \\
d_{\mathrm{k}_{\mathrm{T}}}^{g} & =C_{A}\left(\frac{67}{9}-\frac{3 \pi^{2}}{4}\right)-T_{F} n_{f}\left(\frac{23}{9}\right) .
\end{aligned}
$$


Note that, the unmeasured jet functions are boost-invariant to all orders in perturbation theory, whereas the jet energy functions are boost-covariant up to terms which are power suppressed in $R$. This gives a strong constraint on the $R$ dependence of the jet and soft functions in the factorization theorem. For example, the $R$ dependence of the unmeasured jet functions can only appear as the logarithms of the ratio between $\omega \tan \frac{R}{2}$ and $\mu$.

The differential jet shapes of quark jets at $\mathcal{O}\left(\alpha_{s}\right)$ are

$$
\begin{aligned}
\psi_{\text {cone }}^{q}(r) & =\frac{\alpha_{s} C_{F}}{2 \pi} \frac{1}{16 \sin r}\left[1-\left(\frac{49+2 X+X^{2}}{(1+X)^{2}}+64 \ln \frac{X}{1+X}\right) \sec ^{2} \frac{r}{2}\right] \\
\psi_{k_{T}}^{q}(r) & =\frac{\alpha_{s} C_{F}}{2 \pi} \frac{1}{\sin r}\left(-3+6 X-3 X^{2}-4 \ln X\right) \sec ^{2} \frac{r}{2},
\end{aligned}
$$

while for gluon jets, we have

$$
\begin{gathered}
\psi_{\text {cone }}^{g}(r)=\frac{\alpha_{s}}{2 \pi} \frac{1}{\sin r}\left[\frac{-\left(11+20 X+12 X^{2}\right) C_{A}+2\left(2+2 X+3 X^{2}\right) T_{F} n_{f}}{3(1+X)^{4}}\right. \\
\left.-4 C_{A} \ln \frac{X}{1+X} \sec ^{2} \frac{r}{2}\right] \\
\psi_{k_{T}}^{g}(r)=\frac{\alpha_{s}}{2 \pi} \frac{1}{\sin r}\left[\left(-\frac{11}{3}+8 X-6 X^{2}+\frac{8 X^{3}}{3}-X^{4}-4 \ln X\right) C_{A}\right. \\
\left.+\left(\frac{4}{3}-4 X+6 X^{2}-\frac{16 X^{3}}{3}+2 X^{4}\right) T_{F} n_{f}\right] .
\end{gathered}
$$

In the $r \ll R \ll 1$ limit, the differential jet shapes become

$$
\begin{aligned}
\psi^{q}(r) & =\frac{\alpha_{s} C_{F}}{2 \pi} \frac{1}{r}\left[4 \ln \frac{R}{r}-3+10(\text { or } 6) \frac{r}{R}\right] \\
\psi^{g}(r) & =\frac{\alpha_{s}}{2 \pi} \frac{1}{r}\left[4 C_{A} \ln \frac{R}{r}-\frac{11}{3} C_{A}+\frac{4}{3} T_{F} n_{f}+\left(12(\text { or } 8) C_{A}-4 T_{F} n_{f}\right) \frac{r}{R}\right],
\end{aligned}
$$

for jets reconstructed using the cone (or anti- $\mathrm{k}_{\mathrm{T}}$ ) algorithm. The difference between the differential jet shapes with different jet algorithms is of $\mathcal{O}(r / R)$, which can be important at the periphery of the jet. Note that in the $r \ll R \ll 1$ limit the above results reproduce the differential jet shapes calculated at $\mathcal{O}\left(\alpha_{s}\right)$ in [18].

\subsection{Renormalization-group equations and resummation}

To resum the large logarithms we need to know how the jet energy functions evolve in energy. The renormalization-group equations satisfied by the jet energy functions are the following,

$$
\begin{aligned}
& \frac{d J_{\omega}^{q E_{r}}(\mu)}{d \ln \mu}=\left[-C_{F} \Gamma_{\text {cusp }}\left(\alpha_{s}\right) \ln \frac{\omega^{2} \tan ^{2} \frac{R}{2}}{\mu^{2}}-2 \gamma^{q}\left(\alpha_{s}\right)\right] J_{\omega}^{q E_{r}}(\mu) \\
& \frac{d J_{\omega}^{g E_{r}}(\mu)}{d \ln \mu}=\left[-C_{A} \Gamma_{\text {cusp }}\left(\alpha_{s}\right) \ln \frac{\omega^{2} \tan ^{2} \frac{R}{2}}{\mu^{2}}-2 \gamma^{g}\left(\alpha_{s}\right)\right] J_{\omega}^{g E_{r}}(\mu),
\end{aligned}
$$




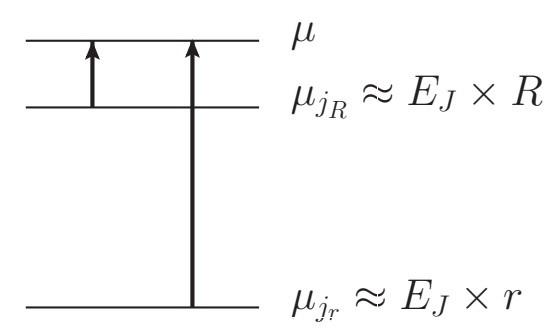

Figure 3. The renormalization group evolution for the jet energy functions. The jet energy functions $J_{\omega}^{E_{r}}(\mu)$ of quark jets and gluon jets are calculated at $\mathcal{O}\left(\alpha_{s}\right)$ at the natural scale $\mu_{j_{r}}$, and they are evolved to a common renormalization scale $\mu$. At the natural scale there are no large logarithms in the jet energy functions. Large logarithms of the form $\log r / R$ in jet shapes are resummed by the renormalization group evolution between the two jet scales $\mu_{j_{r}}$ and $\mu_{j_{R}}$.

where Casimir scaling is assumed to hold up to three loops and $\Gamma_{\text {cusp }}$ is the cusp anomalous dimension. The anomalous dimensions $\Gamma_{\text {cusp }}$ and $\gamma$ can be computed order by order in perturbation theory as series in $\frac{\alpha_{s}}{4 \pi}$,

$$
\begin{aligned}
\Gamma_{\text {cusp }}\left(\alpha_{s}\right) & =\left(\frac{\alpha_{s}}{4 \pi}\right) \Gamma_{0}+\left(\frac{\alpha_{s}}{4 \pi}\right)^{2} \Gamma_{1}+\cdots, \\
\gamma\left(\alpha_{s}\right) & =\left(\frac{\alpha_{s}}{4 \pi}\right) \gamma_{0}+\left(\frac{\alpha_{s}}{4 \pi}\right)^{2} \gamma_{1}+\cdots .
\end{aligned}
$$

The cusp anomalous dimension has been calculated to three loops, and $\gamma\left(\alpha_{s}\right)$ has only been calculated at one loop for both quark and gluon jet energy functions (and unmeasured jet functions),

$$
\gamma_{0}^{q}=-3 C_{F}, \gamma_{0}^{g}=-\beta_{0}
$$

The renormalization-group equation can be solved and the jet energy function can be evolved from its natural scale $\mu_{j_{r}}$ to the renormalization scale $\mu$,

$$
J_{\omega}^{i E_{r}}(\mu)=J_{\omega}^{i E_{r}}\left(\mu_{j_{r}}\right) \exp \left[-2 C_{i} S\left(\mu_{j_{r}}, \mu\right)+2 A_{i}\left(\mu_{j_{r}}, \mu\right)\right]\left(\frac{\omega^{2} \tan ^{2} \frac{R}{2}}{\mu_{j_{r}}^{2}}\right)^{C_{i} A_{\Gamma}\left(\mu_{j_{r}}, \mu\right)}
$$

where $i=q, g$ with $C_{q}=C_{F}$ and $C_{g}=C_{A}$ the Casimir operators of the fundamental and adjoint representations in QCD. Here

$$
S(\nu, \mu)=-\int_{\alpha_{s}(\nu)}^{\alpha_{s}(\mu)} d \alpha \frac{\Gamma_{\text {cusp }}(\alpha)}{\beta(\alpha)} \int_{\alpha_{s}(\nu)}^{\alpha} \frac{d \alpha^{\prime}}{\beta\left(\alpha^{\prime}\right)},
$$

and

$$
A_{i}(\nu, \mu)=-\int_{\alpha_{s}(\nu)}^{\alpha_{s}(\mu)} d \alpha \frac{\gamma^{i}(\alpha)}{\beta(\alpha)}, \quad A_{\Gamma}(\nu, \mu)=-\int_{\alpha_{s}(\nu)}^{\alpha_{s}(\mu)} d \alpha \frac{\Gamma_{\text {cusp }}(\alpha)}{\beta(\alpha)}
$$



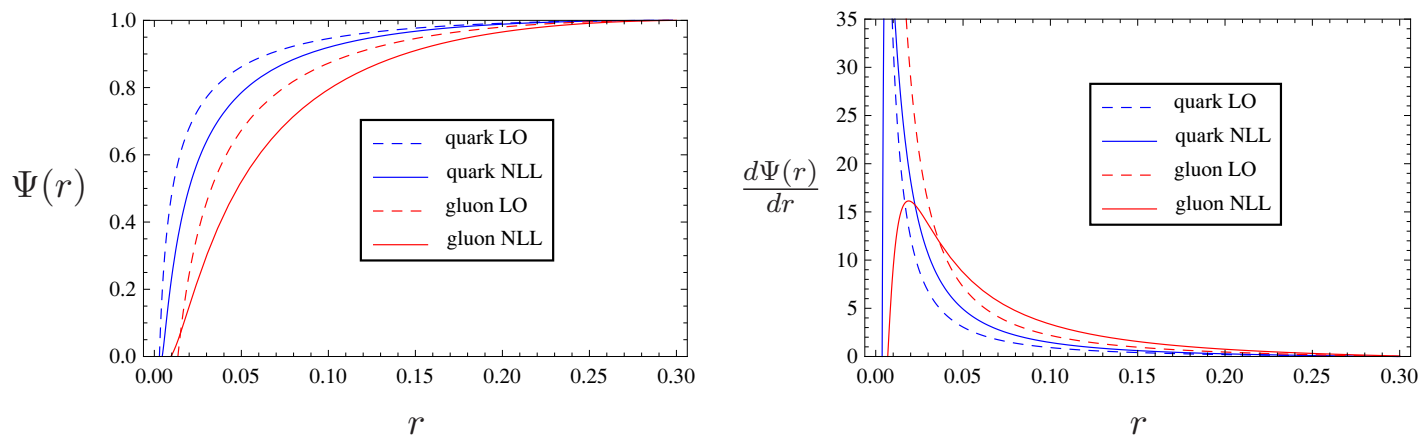

Figure 4. The integral (left) and differential (right) jet shapes for quark and gluon jets reconstructed using the anti- $\mathrm{k}_{\mathrm{T}}$ algorithm with $R=0.3$, with a fixed jet energy $E_{J}=100 \mathrm{GeV}$ plotted as an illustration. The dashed lines are the SCET calculations at leading-order (LO), whereas the solid lines are the ones at next-to-leading logarithmic order (NLL).

are the renormalization-group evolution kernels in SCET. From these, the integral jet shape becomes

$$
\begin{aligned}
\Psi_{\omega}^{i}(r)=\frac{J_{\omega}^{i E_{r}}(\mu)}{J_{\omega}^{i E_{R}}(\mu)}= & \frac{J_{\omega}^{i E_{r}}\left(\mu_{j_{r}}\right)}{J_{\omega}^{i E_{R}}\left(\mu_{j_{R}}\right)} \exp \left[-2 C_{i} S\left(\mu_{j_{r}}, \mu_{j_{R}}\right)+2 A_{i}\left(\mu_{j_{r}}, \mu_{j_{R}}\right)\right] \\
& \times\left(\frac{\mu_{j_{r}}^{2}}{\omega^{2} \tan ^{2} \frac{R}{2}}\right)^{C_{i} A_{\Gamma}\left(\mu_{j_{R}}, \mu_{j_{r}}\right)}
\end{aligned}
$$

Formally $\Psi_{\omega}(r)$ is independent of $\mu, \mu_{j_{r}}$ and $\mu_{j_{R}}$ to all orders in perturbation theory. However, practically we truncate the series at finite order and this induces a renormalization scale dependence. By choosing

$$
\mu_{j_{r}}=\omega \tan \frac{r}{2} \approx E_{J} \times r, \quad \mu_{j_{R}}=\omega \tan \frac{R}{2} \approx E_{J} \times R,
$$

which eliminate large logarithms in the fixed order calculations of $J_{\omega}^{i E_{r}}\left(\mu_{j_{r}}\right)$ and $J_{\omega}^{i E_{R}}\left(\mu_{j_{R}}\right)$ at one loop, ${ }^{5}$ we can resum large logarithms of the form $\ln r / R$ in jet shapes by the renormalization group evolution of the jet energy functions between $\mu_{j_{r}}$ and $\mu_{j_{R}}$ (figure 3 ). The theoretical uncertainties, which come from neglecting higher order terms, can be estimated by exploiting the scale dependence in the resummed results.

\section{Results}

We will compare our calculations with the PYTHIA 8 simulations and the CMS measurements of differential jet shapes in proton-proton collisions with nucleon-nucleon center of mass energy at $\sqrt{s_{\mathrm{NN}}}=2.76 \mathrm{TeV}[26]$. This is the reference for the studies of the jet shape modification in lead-lead collisions, which we will discuss in a forthcoming paper.

\footnotetext{
${ }^{5}$ At two loops, due to the potential issue of non-global logarithms, large logarithms in the fixed order calculations of jet energy functions may not be entirely eliminated with this scale choice. This limits the logarithmic accuracy of our resummed series to NLL.
} 

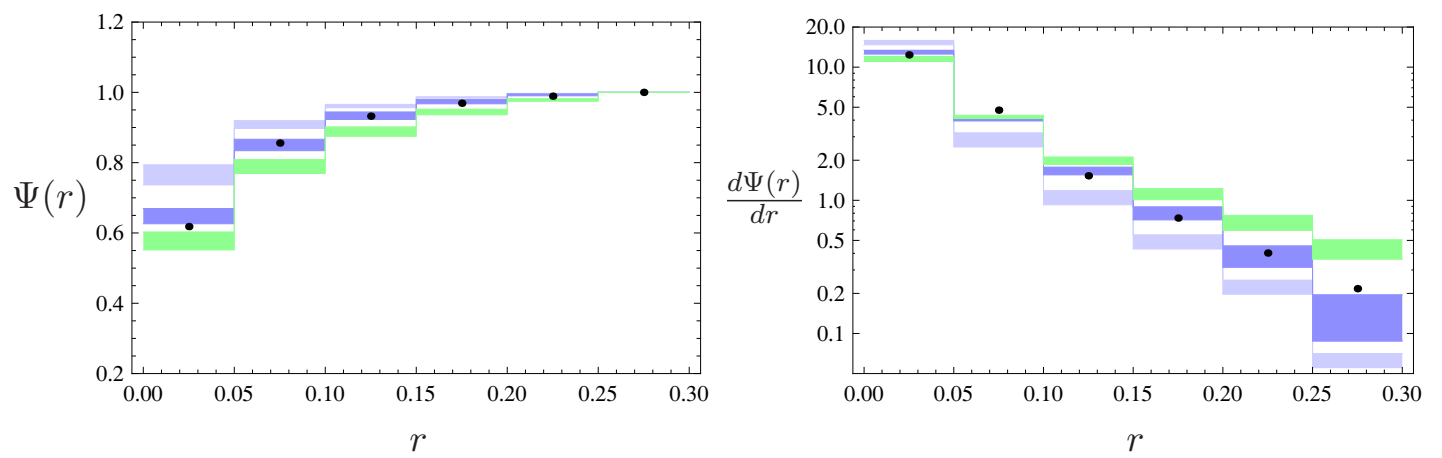

Figure 5. The integral (left) and differential (right) jet shapes in proton-proton collisions with center of mass energy at $\sqrt{s_{\mathrm{NN}}}=2.76 \mathrm{TeV}$ are plotted as a function of $r$, which is the angle from the jet axis. Jets are reconstructed using the anti- $\mathrm{k}_{\mathrm{T}}$ algorithm with $R=0.3$. The cuts on the transverse momenta and rapidity of the jets $\left(p_{T}^{\text {jet }}>100 \mathrm{GeV}\right.$ and $0.3<\left|y^{\text {jet }}\right|<2$ ) are imposed. The dots are the CMS data with negligible experimental uncertainties. The shaded blue boxes are the LO (light) and NLL (dark) results for anti- $\mathrm{k}_{\mathrm{T}}$ jets, with the theoretical uncertainties estimated by varying the jet scales between $\frac{1}{2} \mu_{j_{R}}<\mu<2 \mu_{j_{R}}$. As we can see, the NLL results agree with the data much better than the LO results. The shaded green boxes are the NLL results for cone jets, plotted as an illustration of the algorithm dependence in jet shapes.

Events with dijet production are the most dominant events in the experiment. Jets are reconstructed using the anti- $\mathrm{k}_{\mathrm{T}}$ algorithm with $R=0.3$. This relatively small value of $R$ is chosen to reduce the background fluctuations in heavy ion collisions. The following cuts on the transverse momentum and pseudo-rapidity of a jet are imposed,

$$
p_{T}^{\text {jet }}>100 \mathrm{GeV}, 0.3<\left|y^{\text {jet }}\right|<2 .
$$

The region $\left|y^{\text {jet }}\right|<0.3$ is excluded because of the techniques used in the background subtraction. Note that, the differential jet shapes measured by CMS are constructed from the transverse momenta of the charged particles with $p_{T}^{\text {track }}>1 \mathrm{GeV}$,

$$
\frac{\Delta \Psi(r)}{\Delta r}=\frac{1}{N_{J}} \sum_{J=1}^{N_{J}} \frac{\Psi_{J}^{\mathrm{track}}(r+\delta r / 2)-\Psi_{J}^{\mathrm{track}}(r-\delta r / 2)}{\delta r},
$$

and the jet cone is divided into six annuli between $0<r<0.3$ with $\delta r=0.05$. The difference with the differential jet shapes we calculate is power suppressed by $\mathcal{O}(r / R)$.

To look into the $p_{T}$ dependence of jet shape more exclusively, we also compare our calculations with the CMS differential jet shape measurement for $R=0.7$ anti- $\mathrm{k}_{\mathrm{T}}$ jets in proton-proton collisions at $\sqrt{s}=7 \mathrm{TeV}$ [54]. Central jets with $\left|y^{\text {jet }}\right|<1$ are divided into many $p_{T}$ bins, covering a wide range from $20 \mathrm{GeV}$ to $1 \mathrm{TeV}$, and the jet cone is divided into seven annuli between $0<r<0.7$ with $\delta r=0.1$. The comparison with the first jet shape measurement at the LHC by the ATLAS collaboration [55] gives a similar result.

For the jet production cross section calculations, we use the CTEQ5M parton distribution functions (PDFs) [56] and the leading order $\mathcal{O}\left(\alpha_{s}^{2}\right)$ QCD results. We then average the jet shapes calculated in SCET with the fixed order QCD differential jet production cross section formula. In the SCET calculations of the differential jet shapes $\Psi_{\omega}(r)$, we 

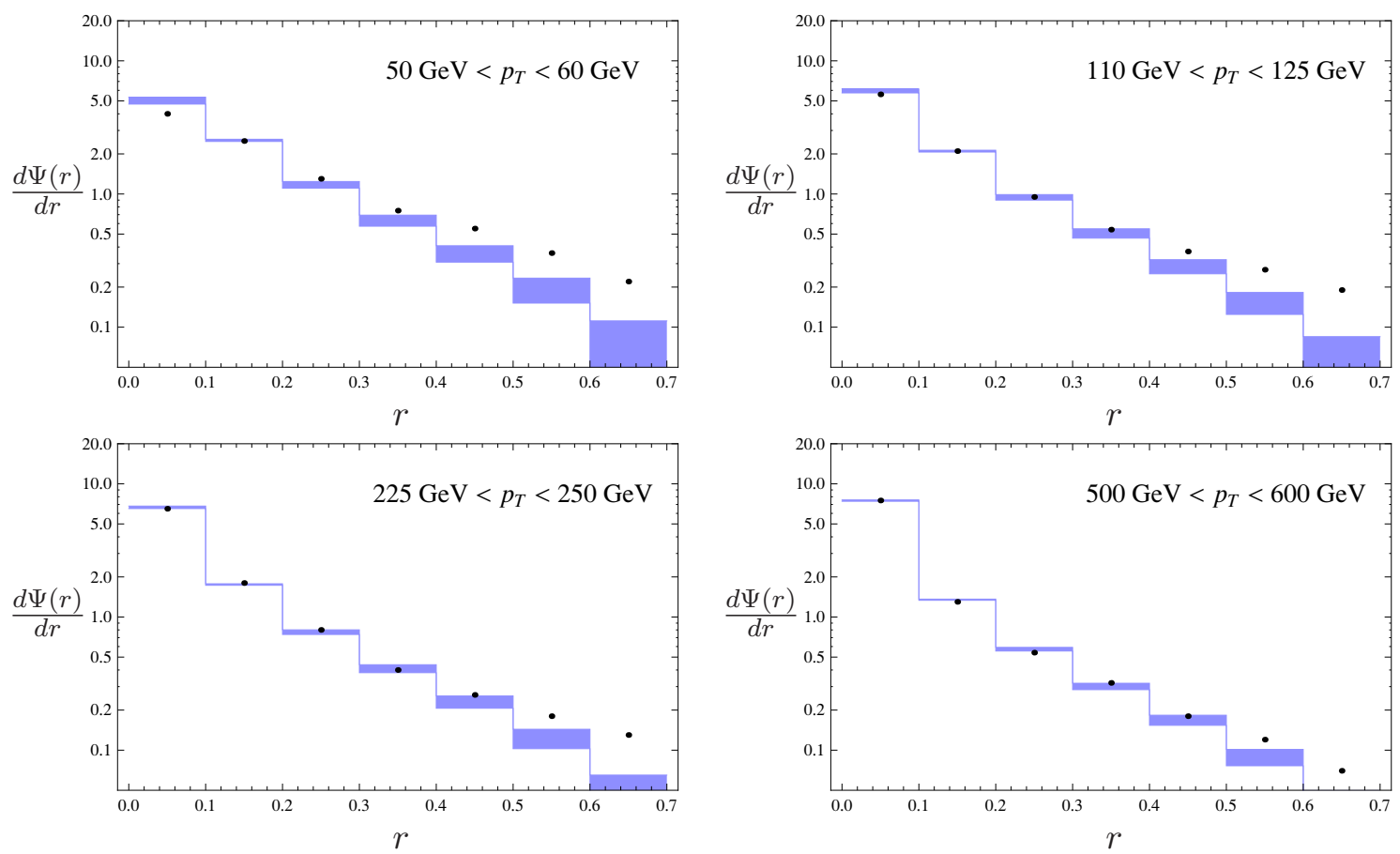

Figure 6. The differential jet shapes in proton-proton collisions at $\sqrt{s}=7 \mathrm{TeV}$. Jets are reconstructed using the anti- $\mathrm{k}_{\mathrm{T}}$ algorithm with $R=0.7$, and the shapes for central jets with $\left|y^{\text {jet }}\right|<1$ are examined in different $p_{T}$ bins: $50 \mathrm{GeV}<p_{T}<60 \mathrm{GeV}, 110 \mathrm{GeV}<p_{T}<125 \mathrm{GeV}$, $225 \mathrm{GeV}<p_{T}<250 \mathrm{GeV}$ and $500 \mathrm{GeV}<p_{T}<600 \mathrm{GeV}$, shown as examples. The dots are the CMS data with negligible experimental uncertainties. The shaded blue boxes are the NLL results, with the theoretical uncertainties estimated by varying the jet scales between $\frac{1}{2} \mu_{j_{R}}<\mu<2 \mu_{j_{R}}$. For high $p_{T}$ jets the calculations reproduce the peak region $(r \ll R)$ very well, with some discrepancy with the data in the tail region $(r \approx R)$ due to power corrections. For low $p_{T}$ jets the power corrections become more significant.

include the one-loop jet energy functions, the two-loop cusp anomalous dimensions $\left(\Gamma_{0}\right.$ and $\left.\Gamma_{1}\right)$ and the one-loop anomalous dimensions $\left(\gamma_{0}^{q, g}\right)$ of the jet energy functions, as well as the two-loop running of the strong coupling constant with $\alpha_{s}\left(m_{Z}\right)=0.1172$ [10]. This will give us the precision formally at next-to-leading logarithmic order (NLL), including terms down to $\alpha_{s}^{n} \ln ^{2 n-1} \frac{r}{R}$. At next-to-next-to-leading logarithmic order (NNLL), there are potential issues related to non-global logarithms which may not be resummed using our renormalization-group technique. Note that, unlike the non-global logarithms which arise from the existence of multiple soft scales when we consider exclusive observables sensitive to soft radiation, in this case non-global logarithms may exist within a SCET collinear sector, which is interesting. We will leave the study of non-global logarithms in jet shapes for future work. ${ }^{6}$

\footnotetext{
${ }^{6}$ While our main focus is on the phenomenological studies of jet shapes in proton-proton and heavy ion collisions, in $e^{+} e^{-}$collisions the full fixed order QCD calculations at NLO [57] and NNLO [58, 59] are available. The coefficients of the leading non-global logarithms at five loops were also recently calculated in the large $N_{c}$ limit [60]. They provide useful information about non-global logarithms and allow checks for precision hadronic observable resummation in $e^{+} e^{-}$collisions beyond NLL.
} 

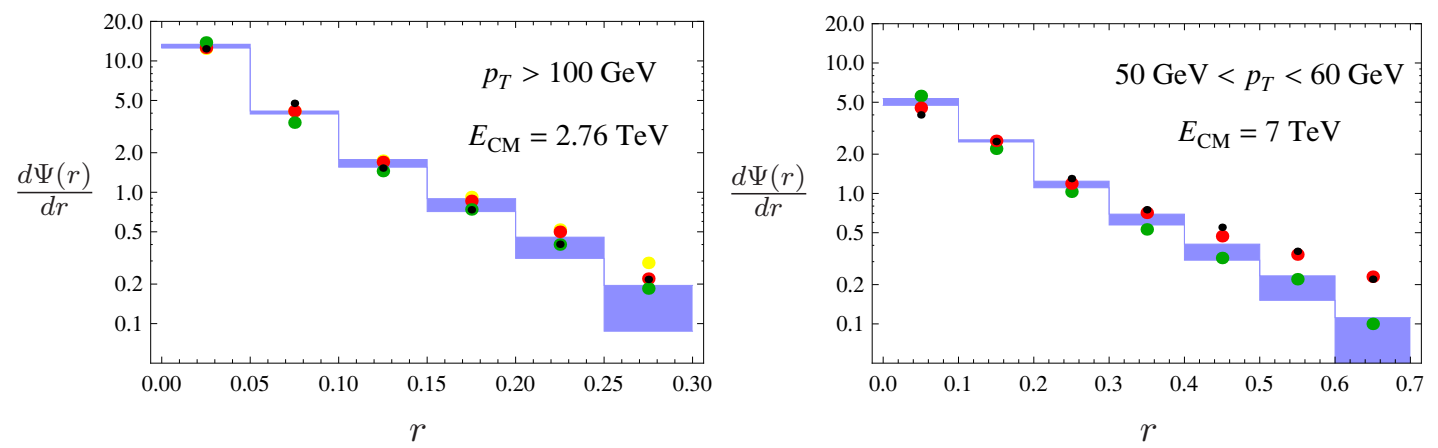

Figure 7. The comparison among the differential jet shapes from the CMS data, the PYTHIA 8 simulations, and the SCET calculations in proton-proton collisions with center of mass energy at 2.76 (left) and 7 (right) TeV. The black dots are the CMS data. The red dots are the PYTHIA simulation with the default tune, while the green dots are the PYTHIA simulation with initial state radiation (ISR) and hadronization turned off. The yellow dots in the left plot are the PYTHIA simulation without the $p_{T}^{\text {track }}>1 \mathrm{GeV}$ cut and the background subtraction. The shaded blue boxes are the NLL SCET results, which agree with PYTHIA without ISR and hadronization.

Figure 4 shows the integral and differential jet shapes of quark jets and gluon jets calculated at leading-order (LO) and next-to-leading logarithmic order (NLL) in SCET. For illustration we plot the energy distributions for jets with a fixed jet energy at $100 \mathrm{GeV}$. The fixed-order jet shape diverges at $r=0$ due to Sudakov logarithms, which need to be resummed. As we can see from the location of the peaks of the NLL differential jet shape distributions, quark jets are more localized whereas gluon jets are more spread out. Also, the effect of resummation is important throughout the whole range of $r$.

Figure 5 shows the comparison between our LO and NLL calculations and the CMS measurement of the integral and differential jet shapes in proton-proton collisions at $\sqrt{s_{\mathrm{NN}}}=2.76 \mathrm{TeV}$. Jets are reconstructed using the anti- $\mathrm{k}_{\mathrm{T}}$ algorithm with a small $R=0.3$. This is the reference for the studies of the jet shape modification in heavy ion collisions. The data are shown as the dots in the plot with negligible experimental uncertainties, which demands precise theoretical calculations. The shaded boxes are the theoretical uncertainties we estimate by varying the jet scales between $\frac{1}{2} \mu_{j_{R}}<\mu<2 \mu_{j_{R}}$. Note that the distribution is plotted with the logarithmic scale in the vertical axis. The LO calculation, due to its divergent nature, certainly can not describe the data and resummation becomes necessary. The results for cone jets are also shown to illustrate the algorithm dependence in jet shapes. Note that cone jets are more spread out than anti- $\mathrm{k}_{\mathrm{T}}$ jets.

Figure 6 shows a similar comparison of our calculations with the CMS differential jet shape measurement in proton-proton collisions at $\sqrt{s}=7 \mathrm{TeV}$. Here, jets are reconstructed using the anti- $\mathrm{k}_{\mathrm{T}}$ algorithm with a larger $R=0.7$, and only the central jets with $\left|y^{\text {jet}}\right|<1$ are considered. We examine the differential jet shapes for jets in different $p_{T}$ bins: $50 \mathrm{GeV}<p_{T}<60 \mathrm{GeV}, 110 \mathrm{GeV}<p_{T}<125 \mathrm{GeV}, 225 \mathrm{GeV}<p_{T}<250 \mathrm{GeV}$ and $500 \mathrm{GeV}<p_{T}<600 \mathrm{GeV}$, as examples. Again the dots are the CMS data with negligible experimental uncertainties, and the theoretical uncertainties are estimated by scale variation. As we can see, for high $p_{T}$ jets the calculations reproduce the peak region $(r \ll R)$ 
very well, with some discrepancy with the data in the tail region $(r \approx R)$ due to the power corrections of $\mathcal{O}(R)$. For low $p_{T}$ jets the power corrections of $\mathcal{O}(\Lambda / Q)$ become more significant because a considerable amount of radiation is outside the jets, which makes the jet more spread out. Also, this is the region where initial state radiation and non-perturbative effects also become significant.

Figure 7 shows the comparison of the jet shapes obtained in this work with PYTHIA 8 simulations at center of mass energies $2.76 \mathrm{TeV}$ and $7 \mathrm{TeV}$. We turn on and off the contributions from initial state radiation (ISR) and hadronization to study their effects. The SCET calculations ignore ISR and hadronization, and they agree well with the PYTHIA simulation without these effects. PYTHIA with the default tune also reasonably agrees with the data. Note that for the jet shapes in the $2.76 \mathrm{TeV}$ collisions, there are several caveats in comparing the data with the SCET resummed results. The jet shapes are reconstructed using only the charged particles with the $p_{T}^{\text {track }}>1 \mathrm{GeV}$ cut. Also, to deal with the huge underlying event contamination in heavy ion collisions, an $\eta$-reflected background subtraction is performed also in reconstructing the jet shapes in proton collisions. These may affect the tail of the jet shape at about 10 to $20 \%$ level.

\section{Summary and discussion}

In this paper we calculated the integral and differential jet shapes in proton-proton collisions at the LHC using soft-collinear effective theory (SCET). We performed resummation at next-to-leading logarithmic (NLL) accuracy, neglecting contributions from initial state radiation and non-perturbative effects. We aimed at obtaining a simple factorized form for the jet shape. Once we choose a recoil-free jet axis, which always points in the collinear momentum direction, the factorized expression assumes a product form, which allows for the cancelation of the hard, unmeasured jet and soft functions in the calculation. The integral jet shape is then a ratio between two jet energy functions which we calculate at leading order (LO) for both quark jets and gluon jets reconstructed using the cone or the anti- $\mathrm{k}_{\mathrm{T}}$ algorithm.

We compared our NLL calculation with the PYTHIA 8 simulation and the CMS measurement of jet shapes in proton-proton collisions at both $\sqrt{s}=2.76 \mathrm{TeV}$ and $\sqrt{s}=7 \mathrm{TeV}$ and found good agreement. This sets the baseline calculation for the study of the jet shape modification in heavy ion collisions, which we will discuss in a forthcoming paper. We showed that the LO calculation can not describe the data well and that resummation is essential. By examining the jet shapes for jets with different transverse momenta, we found that for low $p_{T}$ jets the power corrections are significant. Physically, this is the region where initial-state radiation and non-perturbative effects play a role. For high $p_{T}$ jets the NLL resummed differential jet shape agrees with the data very well in the peak region with some room for power corrections at the very periphery of the jet.

To go beyond this precision systematically, at next-to-next-to-leading logarithmic (NNLL) accuracy we will need to calculate the two-loop jet energy function and its twoloop anomalous dimension. At this order issues about non-global logarithms and the way to resum them will also arise. The jet algorithm dependence will become more interesting 
because at this order we can distinguish between different recombination algorithms. It would be interesting to calculate the jet energy function, as well as the unmeasured jet function at two loops to investigate these questions. The boost properties of these jet functions can also allow us to constrain the $\log R$ dependence. On the other hand, even though the soft function cancels in the jet shape calculation, it is of importance for the resummation of the jet rate. It would be interesting to obtain the two-loop soft function, which has been calculated for a more complicated situation of the jet thrust [61, 62]. This simpler exercise will allow us to check the consistency of the factorization theorem of jet rate and give us insight of the possible refactorization of the soft sector without the complication of the extra measurements.

\section{Acknowledgments}

Y.-T. C. would like to thank Andrew Hornig, Andrew Larkoski, Christopher Lee, Yen-Jie Lee, Hsiang-nan Li, Yaxian Mao, Matthew Schwartz and Wouter Waalewijn for very helpful discussions and comments on the manuscript. The authors would also like to thank the anonymous referee for careful review of the paper. Y.-T. Chien and I. Vitev are supported by the US Department of Energy, Office of Science.

Open Access. This article is distributed under the terms of the Creative Commons Attribution License (CC-BY 4.0), which permits any use, distribution and reproduction in any medium, provided the original author(s) and source are credited.

\section{References}

[1] E. Farhi, A QCD test for jets, Phys. Rev. Lett. 39 (1977) 1587 [INSPIRE].

[2] H. Georgi and M. Machacek, A simple QCD prediction of jet structure in $e^{+} e^{-}$annihilation, Phys. Rev. Lett. 39 (1977) 1237 [INSPIRE].

[3] G.C. Fox and S. Wolfram, Observables for the analysis of event shapes in $e^{+} e^{-}$annihilation and other processes, Phys. Rev. Lett. 41 (1978) 1581 [INSPIRE].

[4] C.L. Basham, L.S. Brown, S.D. Ellis and S.T. Love, Energy correlations in electron-positron annihilation: testing QCD, Phys. Rev. Lett. 41 (1978) 1585 [INSPIRE].

[5] C.L. Basham, L.S. Brown, S.D. Ellis and S.T. Love, Energy correlations in electron-positron annihilation in quantum chromodynamics: asymptotically free perturbation theory, Phys. Rev. D 19 (1979) 2018 [InSPIRE].

[6] ALEPH collaboration, A. Heister et al., Studies of $Q C D$ at $e^{+} e^{-}$centre-of-mass energies between $91 \mathrm{GeV}$ and $209 \mathrm{GeV}$, Eur. Phys. J. C 35 (2004) 457 [InSPIRE].

[7] DELPHI collaboration, J. Abdallah et al., A study of the energy evolution of event shape distributions and their means with the DELPHI detector at LEP, Eur. Phys. J. C 29 (2003) 285 [hep-ex/0307048] [INSPIRE].

[8] L3 collaboration, P. Achard et al., Studies of hadronic event structure in $e^{+} e^{-}$annihilation from $30 \mathrm{GeV}$ to $209 \mathrm{GeV}$ with the L3 detector, Phys. Rept. 399 (2004) 71 [hep-ex/0406049] [INSPIRE]. 
[9] OPAL collaboration, G. Abbiendi et al., Measurement of event shape distributions and moments in $e^{+} e^{-} \rightarrow$ hadrons at $91 \mathrm{GeV}-209 \mathrm{GeV}$ and a determination of $\alpha_{s}$,

Eur. Phys. J. C 40 (2005) 287 [hep-ex/0503051] [INSPIRE].

[10] T. Becher and M.D. Schwartz, A precise determination of $\alpha_{s}$ from LEP thrust data using effective field theory, JHEP 07 (2008) 034 [arXiv:0803.0342] [INSPIRE].

[11] Y.-T. Chien and M.D. Schwartz, Resummation of heavy jet mass and comparison to LEP data, JHEP 08 (2010) 058 [arXiv:1005.1644] [INSPIRE].

[12] R.A. Davison and B.R. Webber, Non-perturbative contribution to the thrust distribution in $e^{+} e^{-}$annihilation, Eur. Phys. J. C 59 (2009) 13 [arXiv:0809.3326] [INSPIRE].

[13] R. Abbate, M. Fickinger, A.H. Hoang, V. Mateu and I.W. Stewart, Thrust at $N^{3} L L$ with power corrections and a precision global fit for $\alpha_{m Z}$, Phys. Rev. D 83 (2011) 074021 [arXiv: 1006.3080] [INSPIRE].

[14] A. Altheimer et al., Boosted objects and jet substructure at the LHC. Report of BOOST2012, held at IFIC Valencia, 23rd-27th of July 2012, Eur. Phys. J. C 74 (2014) 2792 [arXiv:1311.2708] [INSPIRE].

[15] J. Gallicchio and M.D. Schwartz, Quark and gluon tagging at the LHC, Phys. Rev. Lett. 107 (2011) 172001 [arXiv:1106.3076] [INSPIRE].

[16] J. Gallicchio and M.D. Schwartz, Quark and gluon jet substructure, JHEP 04 (2013) 090 [arXiv: 1211.7038] [INSPIRE].

[17] S.D. Ellis, Z. Kunszt and D.E. Soper, Jets at hadron colliders at order $\alpha_{s}^{3}$ : a look inside, Phys. Rev. Lett. 69 (1992) 3615 [hep-ph/9208249] [INSPIRE].

[18] M.H. Seymour, Jet shapes in hadron collisions: Higher orders, resummation and hadronization, Nucl. Phys. B 513 (1998) 269 [hep-ph/9707338] [INSPIRE].

[19] H.-n. Li, Z. Li and C.-P. Yuan, QCD resummation for jet substructures, Phys. Rev. Lett. 107 (2011) 152001 [arXiv:1107.4535] [INSPIRE].

[20] H.-n. Li, Z. Li and C.-P. Yuan, QCD resummation for light-particle jets, Phys. Rev. D 87 (2013) 074025 [arXiv: 1206.1344] [INSPIRE].

[21] I. Vitev, S. Wicks and B.-W. Zhang, A theory of jet shapes and cross sections: from hadrons to nuclei, JHEP 11 (2008) 093 [arXiv:0810.2807] [INSPIRE].

[22] Y. He, I. Vitev and B.-W. Zhang, $\mathcal{O}\left(\alpha_{s}^{3}\right)$ analysis of inclusive jet and di-jet production in heavy ion reactions at the Large Hadron Collider, Phys. Lett. B 713 (2012) 224 [arXiv: 1105.2566] [INSPIRE].

[23] ATLAS collaboration, Measurement of the jet radius and transverse momentum dependence of inclusive jet suppression in lead-lead collisions at $\sqrt{s_{N N}}=2.76 \mathrm{TeV}$ with the ATLAS detector, Phys. Lett. B 719 (2013) 220 [arXiv:1208.1967] [INSPIRE].

[24] ATLAS collaboration, Observation of a centrality-dependent dijet asymmetry in lead-lead collisions at $\sqrt{s_{N N}}=2.77 \mathrm{TeV}$ with the ATLAS detector at the LHC, Phys. Rev. Lett. 105 (2010) 252303 [arXiv:1011.6182] [INSPIRE].

[25] CMS collaboration, Observation and studies of jet quenching in $\mathrm{PbPb}$ collisions at nucleon-nucleon center-of-mass energy $=2.76$ TeV, Phys. Rev. C 84 (2011) 024906 [arXiv:1102.1957] [INSPIRE]. 
[26] CMS collaboration, Modification of jet shapes in PbPb collisions at $\sqrt{s_{N N}}=2.76$ TeV, Phys. Lett. B 730 (2014) 243 [arXiv:1310.0878] [InSPIRE].

[27] ATLAS and CMS collaboration, P. Kurt, Jet quenching with ATLAS and CMS, EPJ Web Conf. 66 (2014) 04017.

[28] M. Gyulassy, P. Levai and I. Vitev, Reaction operator approach to nonAbelian energy loss, Nucl. Phys. B 594 (2001) 371 [nucl-th/0006010] [InSPIRE].

[29] M. Gyulassy, P. Levai and I. Vitev, Non-Abelian energy loss at finite opacity, Phys. Rev. Lett. 85 (2000) 5535 [nucl-th/0005032] [INSPIRE].

[30] G.-L. Ma, Medium modifications of jet shapes in Pb+Pb collisions at $\sqrt{s_{N N}}=2.76 \mathrm{TeV}$ within a multiphase transport model, Phys. Rev. C 89 (2014) 024902 [arXiv:1309.5555] [INSPIRE].

[31] R. Perez-Ramos and T. Renk, In-medium jet shape from energy collimation in parton showers: comparison with CMS PbPb data at 2.76 TeV, Phys. Rev. D 90 (2014) 014018 [arXiv: 1401.5283] [INSPIRE].

[32] C.W. Bauer, S. Fleming and M.E. Luke, Summing Sudakov logarithms in $B \rightarrow X(s \gamma)$ in effective field theory, Phys. Rev. D 63 (2000) 014006 [hep-ph/0005275] [INSPIRE].

[33] C.W. Bauer, S. Fleming, D. Pirjol and I.W. Stewart, An effective field theory for collinear and soft gluons: heavy to light decays, Phys. Rev. D 63 (2001) 114020 [hep-ph/0011336] [INSPIRE].

[34] C.W. Bauer and I.W. Stewart, Invariant operators in collinear effective theory, Phys. Lett. B 516 (2001) 134 [hep-ph/0107001] [INSPIRE].

[35] C.W. Bauer, D. Pirjol and I.W. Stewart, Soft collinear factorization in effective field theory, Phys. Rev. D 65 (2002) 054022 [hep-ph/0109045] [INSPIRE].

[36] C.W. Bauer, S. Fleming, D. Pirjol, I.Z. Rothstein and I.W. Stewart, Hard scattering factorization from effective field theory, Phys. Rev. D 66 (2002) 014017 [hep-ph/0202088] [INSPIRE].

[37] A. Idilbi and A. Majumder, Extending soft-collinear-effective-theory to describe hard jets in dense QCD media, Phys. Rev. D 80 (2009) 054022 [arXiv:0808.1087] [InSPIRE].

[38] G. Ovanesyan and I. Vitev, An effective theory for jet propagation in dense QCD matter: jet broadening and medium-induced bremsstrahlung, JHEP 06 (2011) 080 [arXiv:1103.1074] [INSPIRE].

[39] G. Ovanesyan and I. Vitev, Medium-induced parton splitting kernels from soft collinear effective theory with Glauber gluons, Phys. Lett. B 706 (2012) 371 [arXiv:1109.5619] [INSPIRE].

[40] M. Fickinger, G. Ovanesyan and I. Vitev, Angular distributions of higher order splitting functions in the vacuum and in dense QCD matter, JHEP 07 (2013) 059 [arXiv:1304.3497] [INSPIRE].

[41] Z.-B. Kang, R. Lashof-Regas, G. Ovanesyan, P. Saad and I. Vitev, Jet quenching phenomenology from soft-collinear effective theory with Glauber gluons, arXiv:1405.2612 [INSPIRE].

[42] Y.-T. Chien and I. Vitev, Calculating jet shape modifications in heavy ion collisions using soft-collinear effective theory, work in progress. 
[43] L.G. Almeida et al., Comparing and counting logs in direct and effective methods of QCD resummation, JHEP 04 (2014) 174 [arXiv:1401.4460] [INSPIRE].

[44] M. Dasgupta and G.P. Salam, Resummation of nonglobal QCD observables, Phys. Lett. B 512 (2001) 323 [hep-ph/0104277] [INSPIRE].

[45] D. Bertolini, T. Chan and J. Thaler, Jet observables without jet algorithms, JHEP 04 (2014) 013 [arXiv: 1310.7584] [INSPIRE].

[46] A.J. Larkoski, D. Neill and J. Thaler, Jet shapes with the broadening axis, JHEP 04 (2014) 017 [arXiv: 1401.2158] [INSPIRE].

[47] M. Cacciari, G.P. Salam and G. Soyez, The anti-k(t) jet clustering algorithm, JHEP 04 (2008) 063 [arXiv:0802.1189] [INSPIRE].

[48] D. Appell, G.F. Sterman and P.B. Mackenzie, Soft gluons and the normalization of the Drell-Yan cross-section, Nucl. Phys. B 309 (1988) 259 [INSPIRE].

[49] S. Catani, M.L. Mangano and P. Nason, Sudakov resummation for prompt photon production in hadron collisions, JHEP 07 (1998) 024 [hep-ph/9806484] [INSPIRE].

[50] T. Becher, M. Neubert and G. Xu, Dynamical threshold enhancement and resummation in Drell-Yan production, JHEP 07 (2008) 030 [arXiv:0710.0680] [INSPIRE].

[51] Y.-T. Chien, R. Kelley, M.D. Schwartz and H.X. Zhu, Resummation of jet mass at hadron colliders, Phys. Rev. D 87 (2013) 014010 [arXiv: 1208.0010] [INSPIRE].

[52] C.W. Bauer, C. Lee, A.V. Manohar and M.B. Wise, Enhanced nonperturbative effects in Z decays to hadrons, Phys. Rev. D 70 (2004) 034014 [hep-ph/0309278] [INSPIRE].

[53] S.D. Ellis, C.K. Vermilion, J.R. Walsh, A. Hornig and C. Lee, Jet shapes and jet algorithms in SCET, JHEP 11 (2010) 101 [arXiv:1001.0014] [INSPIRE].

[54] CMS collaboration, Shape, transverse size and charged hadron multiplicity of jets in pp collisions at $7 \mathrm{TeV}$, JHEP 06 (2012) 160 [arXiv:1204.3170] [INSPIRE].

[55] ATLAS collaboration, Study of jet shapes in inclusive jet production in pp collisions at $\sqrt{s}=7$ TeV using the ATLAS detector, Phys. Rev. D 83 (2011) 052003 [arXiv:1101.0070] [INSPIRE].

[56] W.K. Tung, H.L. Lai, A. Belyaev, J. Pumplin, D. Stump et al., Heavy Quark Mass Effects in Deep Inelastic Scattering and Global QCD Analysis, JHEP 02 (2007) 053 [hep-ph/0611254] [INSPIRE].

[57] S. Catani and M.H. Seymour, The dipole formalism for the calculation of QCD jet cross-sections at next-to-leading order, Phys. Lett. B 378 (1996) 287 [hep-ph/9602277] [INSPIRE].

[58] A. Gehrmann-De Ridder, T. Gehrmann, E.W.N. Glover and G. Heinrich, Infrared structure of $e^{+} e^{-} \rightarrow 3$ jets at NNLO, JHEP 11 (2007) 058 [arXiv:0710.0346] [INSPIRE].

[59] A. Gehrmann-De Ridder, T. Gehrmann, E.W.N. Glover and G. Heinrich, EERAD3: Event shapes and jet rates in electron-positron annihilation at order $\alpha_{s}^{3}$,

Comput. Phys. Commun. 185 (2014) 3331 [arXiv:1402.4140] [INSPIRE].

[60] M.D. Schwartz and H.X. Zhu, Non-global logarithms at 3 loops, 4 loops, 5 loops and beyond, Phys. Rev. D 90 (2014) 065004 [arXiv:1403.4949] [INSPIRE]. 
[61] R. Kelley, M.D. Schwartz, R.M. Schabinger and H.X. Zhu, Jet mass with a jet veto at two loops and the universality of non-global structure, Phys. Rev. D 86 (2012) 054017 [arXiv:1112.3343] [INSPIRE].

[62] A. von Manteuffel, R.M. Schabinger and H.X. Zhu, The complete two-loop integrated jet thrust distribution in soft-collinear effective theory, JHEP 03 (2014) 139 [arXiv:1309.3560] [INSPIRE]. 